\title{
June 2017 Imaging Case of the Month
}

Michael B. Gotway, MD

Department of Radiology

Mayo Clinic Arizona

Scottsdale, Arizona USA

Clinical History: A 30-year-old woman with no significant past medical history presented with complaints of chronic back pain, partially controlled with Ibuprofen. Recently she began to notice shortness of breath. Frontal and lateral chest radiography (Figure 1) was performed.

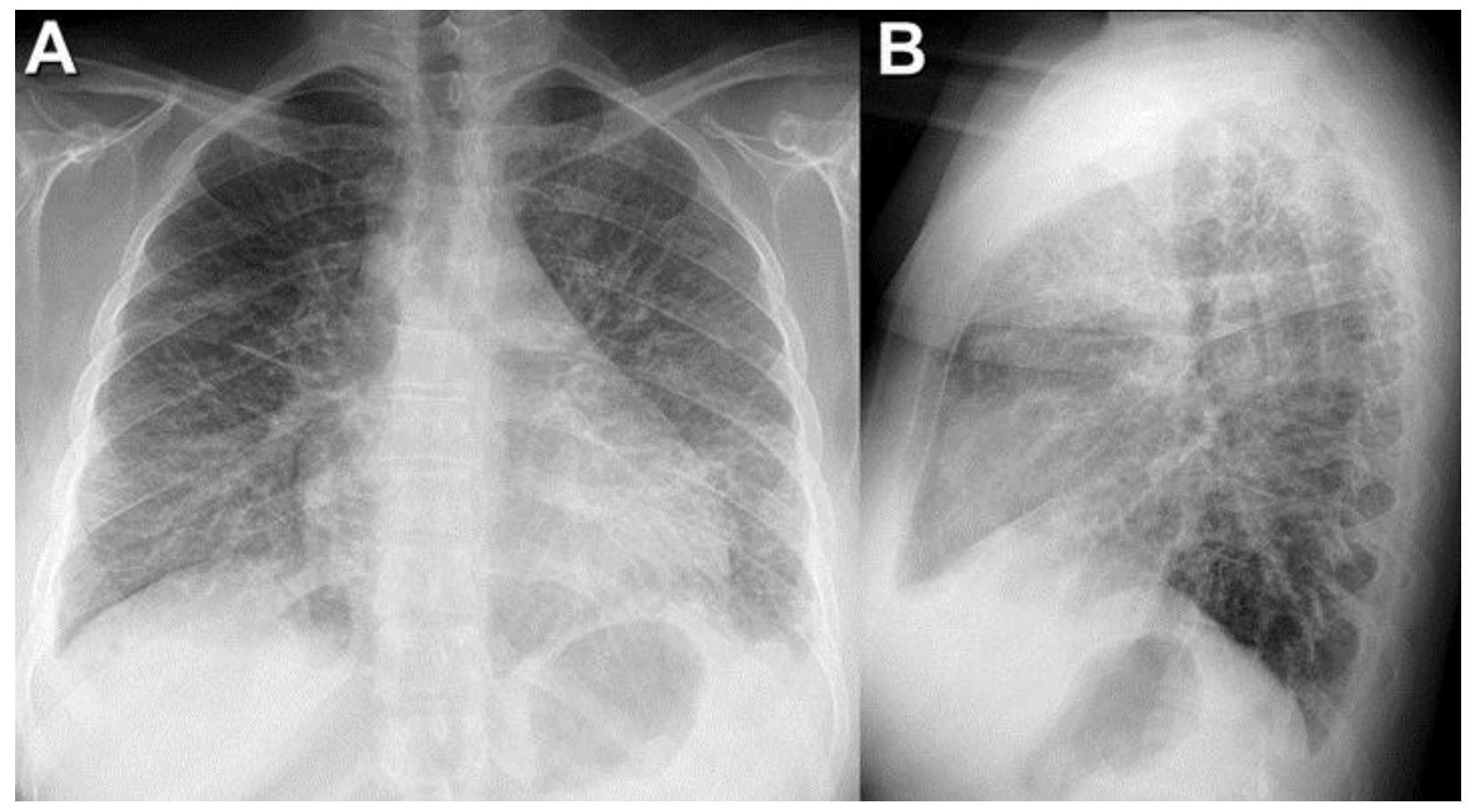

Figure 1. Frontal $(A)$ and lateral $(B)$ chest radiography.

Which of the following statements regarding the chest radiograph is most accurate?

1. The chest radiograph shows a diffuse linear, interstitial pattern

2. The chest radiograph shows a large pleural effusion

3. The chest radiograph shows a mediastinal mass

4. The chest radiograph shows multifocal, bilateral consolidation

5. The chest radiograph shows numerous small nodules 


\section{Correct!}

\section{The chest radiograph shows a diffuse linear, interstitial pattern}

The frontal chest radiograph shows a prominent linear pattern throughout the lungs, involving the central perihilar areas but also quite prominent in the peripheral lung parenchyma. The heart also appears enlarged. No large pleural effusion is present (trace left pleural liquid is possible) and no multifocal consolidation is evident. The widening of the superior mediastinum is vascular in nature; no mediastinal mass is present. A nodular pattern is not evident.

Which of the following statements regarding the chest radiograph is most accurate?

1. The chest radiograph shows interlobular septal thickening

2. The chest radiograph shows Kerley's B lines

3. The chest radiograph shows peripheral basal reticulation

4. 1 and 2

5. 2 and 3 


\section{Correct!}

\section{1 and 2}

The frontal chest radiograph shows a prominent linear pattern throughout the lungs- this linear pattern represents interlobular septal thickening. The interlobular septal thickening is evident in the perihilar regions, but also as 1-2 cm long, perpendicular lines in contact with the peripheral lateral chest wall- this latter description is that of Kerley's B lines. In essence, Kerley's B lines represent peripheral interlobular septal thickening, so both choices $a$ and $b$ are correct. The patterns is a linear one, not a series of "crisscrossing" lines, creating a net-like appearance, so the term "reticulation" here is not an appropriate descriptor.

Which of the following represent the most appropriate differential diagnostic consideration for the chest radiographic pattern present?

1. Chronic hypersensitivity pneumonitis

2. Increased pressure edema

3. Lymphangioleiomyomatosis

4. Streptococcus pneumoniae pneumonia

5. Usual interstitial pneumonia 


\section{Correct! \\ 2. Increased pressure edema}

Given that extensive interlobular septal thickening is present, the one entity among those listed that often results in interlobular septal thickening at thoracic imaging studies is increased pressure edema (also often referred to as hydrostatic pulmonary edema, or less specifically, cardiogenic pulmonary edema). Lymphangioleiomyomatosis (LAM) is a form of cystic lung disease, but occasionally can show a somewhat linear pattern at chest radiography owing to partial visualization of cysts walls; however, even this appearance of LAM is generally not confused with interlobular septal thickening. Usual interstitial pneumonia may occasionally produce some interlobular septal thickening, but the dominant picture is usually that of basal and peripheral reticulation associated with low lung volumes, occasionally even visualization of discrete honeycombing.

Pneumonia due to Streptococcus pneumoniae commonly causes a "lobar pneumonia" pattern, but may also produce segmental consolidation typical of the bronchopneumonia pattern. An "interstitial" appearance for this infection is unusual, and, even when interstitial opacities are present in patients with pneumococcus, the interlobular septal thickening would not be as profound as it is in this patient. The chest radiographic findings of chronic hypersensitivity pneumonitis are ultimately nonspecific, but generally appear as bilateral mid- and upper lung coarse linear and reticular abnormalities, representing fibrosis, associated with areas of architectural distortion, not the fairly diffuse, symmetric interlobular septal thickening, unassociated with distortion, as seen in this patient.

The chest radiographic findings (cardiomegaly and interlobular septal thickening) and patient's new shortness of breath raised the possibility of increased pressure edema, so the patient was begun on diuretic therapy. Chest radiography was repeated 6 days later (Figure 2).

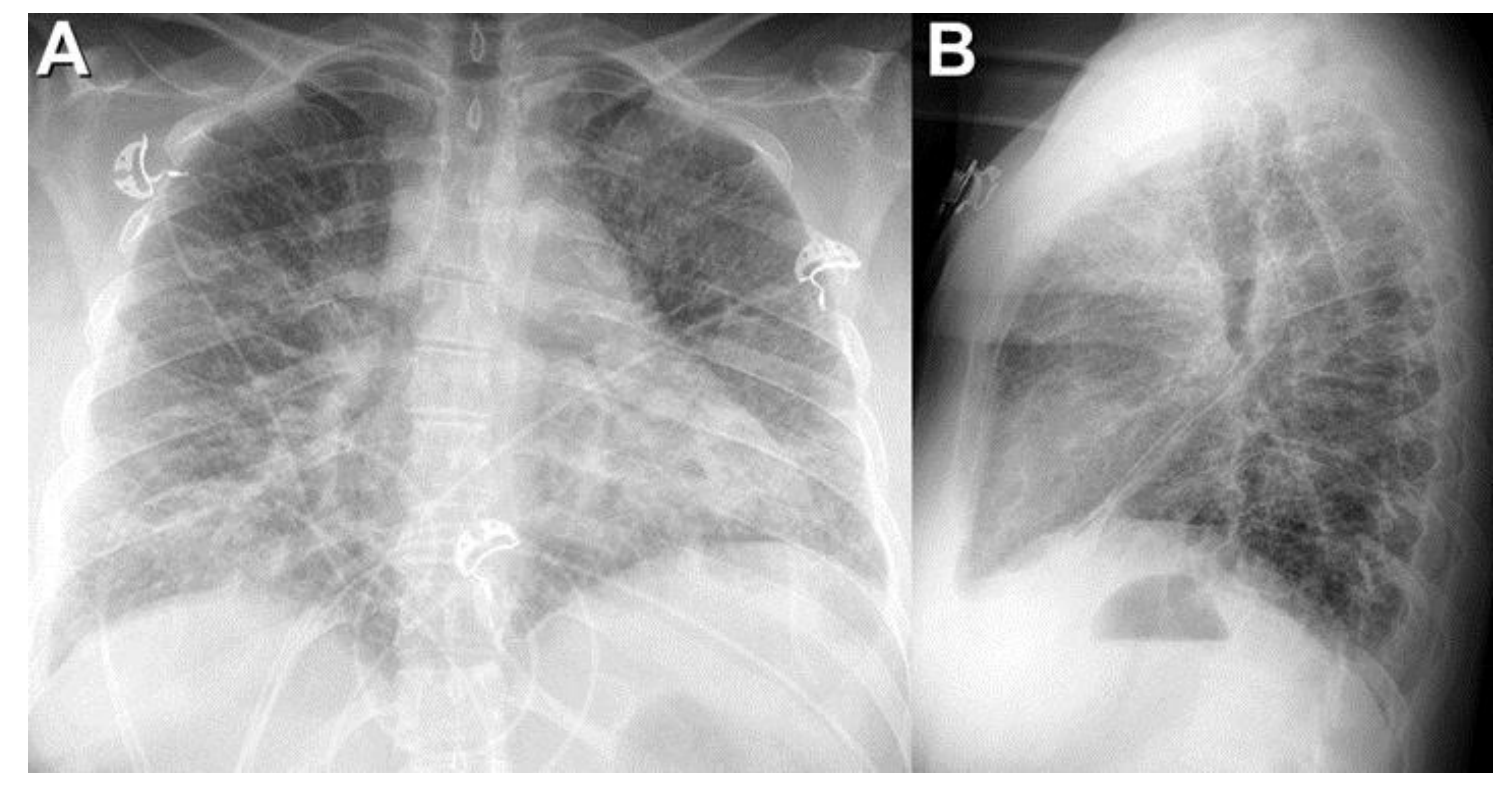

Figure 2. Frontal (A) and lateral (chest radiography performed 6 days after presentation. 
Which of the following represents the most accurate interpretation of the repeat chest radiographic findings?

1. Repeat chest radiography shows enlarging pleural effusions

2. Repeat chest radiography shows the development of pneumothorax

3. The chest radiograph shows new cavitary pulmonary nodules

4. The chest repeat chest radiography shows improvement in the interlobular septal thickening, suggesting improved increased pressure edema

5. The repeat chest radiography shows no change in the interlobular septal thickening, suggesting increased pressure edema is probably not responsible for the chest radiographic findings 


\section{Correct!}

5. The chest repeat chest radiography shows no change in the interlobular septal thickening, suggesting increased pressure edema is probably not responsible for the chest radiographic findings

Repeat chest radiography shows persistent, extensive, bilateral interlobular septal thickening, relatively unchanged since the presentation chest radiograph and apparently unaffected by the attempts at diuresis in the interval. If the interlobular septal thickening were the result of increased pressure edema, some improvement in the chest radiographic findings would be expected following attempts at volume overload correction. Therefore, the absence of such change suggests a cause other than increased pressure edema for the extensive interlobular septal thickening should be sought. No new or enlarging pleural effusions are present and no new cavitary pulmonary nodules are seen. No evidence of pneumothorax is present.

Which of the following represents the next most appropriate step for the evaluation of this patient?

1. ${ }^{18}$ FDG-PET scan

2. ${ }^{68} \mathrm{Ga}$-citrate scan

3. Enhanced thoracic CT

4. Flexible fiberoptic bronchoscopy with transbronchial biopsy

5. Percutaneous transthoracic needle biopsy 


\section{Correct! \\ 3. Enhanced thoracic CT}

Enhanced thoracic CT would provide an excellent means for characterization of the patient's chest radiographic findings. Flexible fiberoptic bronchoscopy with transbronchial biopsy is not a "wrong" answer, in that this technique could provide useful diagnostic information in this patient with extensive interstitial thickening. However, the procedure is premature at this point- further non-invasive characterization could provide information that alters the subsequent management options for this patient. While diffuse pulmonary parenchymal abnormalities are present, there is no suitable target for percutaneous transthoracic lung biopsy- this procedure is typically employed to obtain a tissue diagnosis for patient with focal pulmonary diseases, particularly pulmonary nodules. ${ }^{68} \mathrm{Ga}$-citrate scanning is occasionally used for the assessment of diffuse lung disorders, but the result of this examination are rarely diagnostic and the examination is often of limited utility. ${ }^{18 F}$ FDG-PET scanning is also premature at this point, as this imaging modality is most commonly used in the staging of known malignancy or the assessment of indeterminate pulmonary nodules; ${ }^{8 F F D G-~}$ PET does not typically play a role in the assessment of diffuse pulmonary parenchymal abnormalities.

Enhanced thoracic CT (Figure 3) was performed.

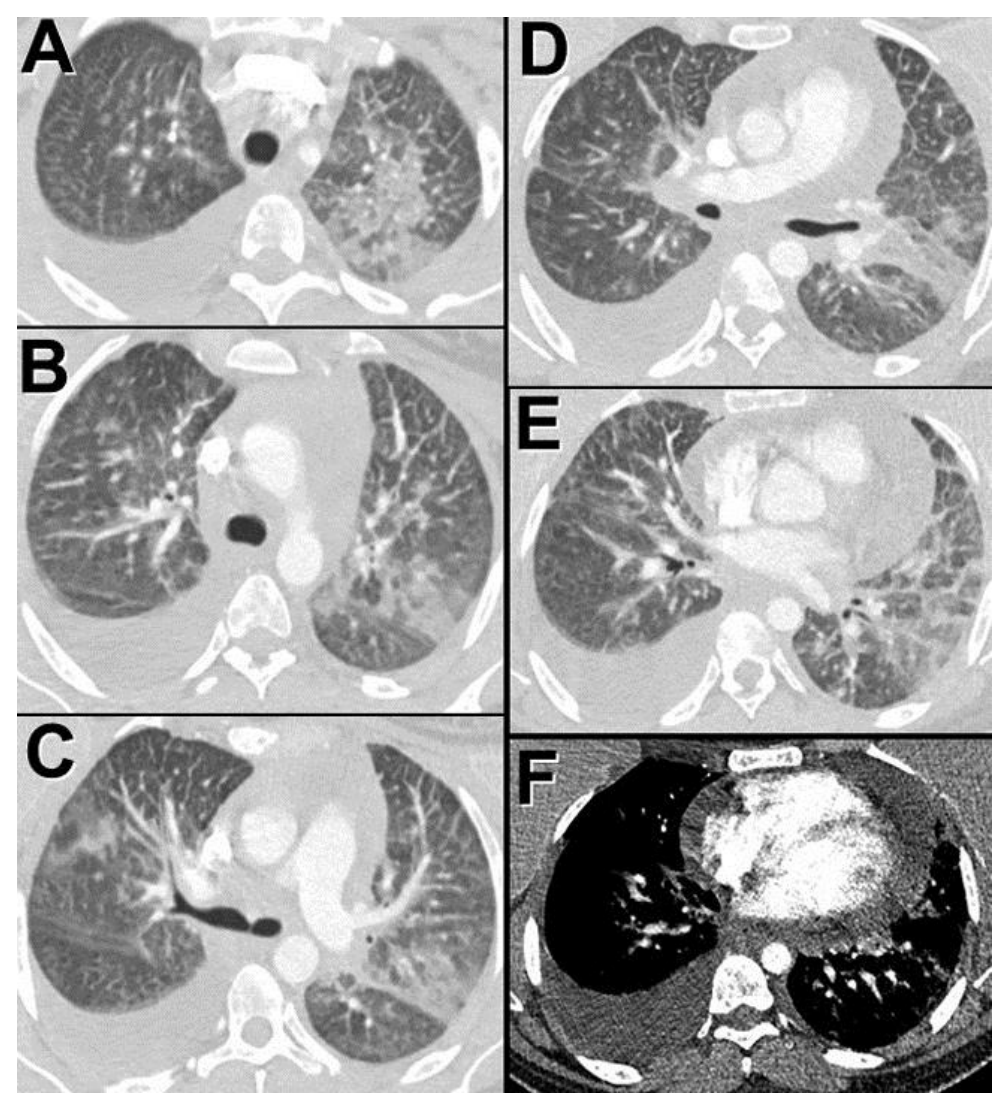

Figure 3. Enhanced thoracic CT displayed in lung (A-E) and soft tissue (F) windows. 
Which of the following statements regarding this imaging study is most accurate?

1. The enhanced thoracic CT shows acute pulmonary embolism

2. The enhanced thoracic CT shows aortic dissection

3. The enhanced thoracic CT shows extensive interlobular septal thickening and pericardial effusion

4. The enhanced thoracic CT shows numerous small pulmonary nodules

5. The enhanced thoracic CT shows pneumothorax 


\section{Correct! \\ 3. The enhanced thoracic CT shows extensive interlobular septal thickening and pericardial effusion}

The enhanced thoracic CT shows extensive interlobular septal thickening, as seen on the two chest radiographs, as well as a moderate-sized pericardial effusion; the latter accounts for the cardiac enlargement seen at chest radiography. Numerous pulmonary nodules are not seen, and there is no evidence of either aortic dissection or acute pulmonary embolism. A right pleural effusion is present, but no pneumothorax is seen.

Transthoracic echocardiography confirmed the presence of pericardial effusion and suggested tamponade physiology. Pericardiocentesis was performed. The fluid was found to be exudative, but infectious disease studies were unrevealing. An extensive histiocytic infiltration on a background of reactive mesothelial cells was noted on cytological examination.

Which of the following represents the next most appropriate step for the evaluation of this patient?
1. Mediastinoscopy
2. Repeat thoracic CT
3. Thoracentesis
4. Thoracic MR
5. Video-assisted thoracoscopic lung biopsy 


\section{Correct! \\ 3. Thoracentesis}

Video-assisted thoracoscopic lung biopsy certainly could obtain a diagnosis in this patient with extensive interstitial abnormalities, but is needlessly invasive at this pointless invasive options, such as flexible fiberoptic bronchoscopy with transbronchial biopsy [this option was not offered in the choices above] and thoracentesis may be performed. Mediastinoscopy is generally reserved for patients with enlarged mediastinal lymph nodes in need of staging for malignancy, or to sample mediastinal masses, and neither are present in this patient. Repeat cross sectional imaging, either CT or MR, is unlikely to provide additional management-altering information to that already known.

Thoracentesis was performed for the right pleural effusion and disclosed an extensive histiocytic infiltration on a background of reactive mesothelial cells; rare atypical epithelioid cells with large, irregular nuclei were seen, but no definite malignancy was present. Percutaneous biopsy of a mildly enlarged retroperitoneal lymph node disclosed only inflammatory tissue and fibroadipose tissue.

The patient underwent bone marrow biopsy which showed metastatic malignancy due to adenocarcinoma with a signet-ring morphology. This morphology promoted a gastrointestinal evaluation and upper endoscopy with biopsy revealed gastric malignancy.

Diagnosis: Pulmonary lymphangitic carcinomatosis due to gastric carcinoma.

\section{References}

1. Colby TV, Swensen SJ. Anatomic distribution and histopathologic patterns in diffuse lung disease: correlation with HRCT. J Thorac Imaging. 1996;11(1):1-26. Erratum in: J Thorac Imaging 1996;11(2):163. [CrossRef] [PubMed]

2. Prakash $P$, Kalra MK, Sharma A, Shepard JA, Digumarthy SR. FDG PET/CT in assessment of pulmonary lymphangitic carcinomatosis. AJR Am J Roentgenol. 2010; 194(1):231-6. [CrossRef] [PubMed]

3. Gruden JF, Webb WR, Naidich DP, McGuinness G. Multinodular disease: anatomic localization at thin-section CT--multireader evaluation of a simple algorithm.

Radiology. 1999;210(3):711-20. [CrossRef] [PubMed]

4. Nishino $\mathrm{M}$, Itoh $\mathrm{H}$, Hatabu $\mathrm{H}$. A practical approach to high-resolution $\mathrm{CT}$ of diffuse lung disease. Eur J Radiol. 2014;83(1):6-19. [CrossRef] [PubMed] 